\title{
Lyapunov Exponents from Kinetic Theory for a Dilute, Field-Driven Lorentz Gas
}

\author{
H. van Beijeren, ${ }^{1}$ J. R. Dorfman, ${ }^{2}$ E. G. D. Cohen, ${ }^{3}$ H. A. Posch, ${ }^{4}$ and Ch. Dellago ${ }^{4}$ \\ ${ }^{1}$ Institute for Theoretical Physics, University of Utrecht, Postbus 80006, 3508 TA Utrecht, The Netherlands \\ ${ }^{2}$ Institute for Physical Science and Technology and Department of Physics, University of Maryland, College Park, Maryland 20742 \\ ${ }^{3}$ The Rockefeller University, 1230 York Avenue, New York, New York 10021 \\ ${ }^{4}$ Institut für Experimentalphysik, Universität Wien, Boltzmanngasse 5, A-1090 Wien, Austria
}

(Received 8 May 1996)

\begin{abstract}
Positive and negative Lyapunov exponents for a dilute, random, two-dimensional Lorentz gas in an applied field, $\vec{E}$, in a steady state at constant energy are computed to order $E^{2}$. The results are $\lambda_{ \pm}=\lambda_{ \pm}^{0}-a_{ \pm}(q E / m v)^{2} t_{0}$ where $\lambda_{ \pm}^{0}$ are the exponents for the field-free Lorentz gas, $a_{+}=11 / 48$, $a_{-}=7 \overline{4} 8, t_{0}$ is the mean free time between collisions, $q$ is the charge, $m$ is the mass, and $v$ is the speed of the particle. The calculation is based on an extended Boltzmann equation in which a radius of curvature, characterizing the separation of two nearby trajectories, is one of the variables in the distribution function. The analytical results are in excellent agreement with computer simulations. [S0031-9007(96)01100-3]
\end{abstract}

PACS numbers: 05.45.+b, 05.20.Dd

One of the outstanding problems in transport theory is to relate macroscopic quantities such as transport coefficients to microscopic quantities that characterize the chaotic dynamics of the system. This chaotic dynamics is responsible for the stochasticlike behavior which leads to normal hydrodynamic processes taking place in fluids. One approach is to consider the system as a Hamiltonian system, obeying classical mechanics, and to apply the escape-rate formalism which can be used to express transport coefficients in terms of Lyapunov exponents and Kolmogorov-Sinai entropies for trajectories in phase space on an appropriate fractal repeller [1]. Another approach to this problem is to consider driven, thermostated systems in a nonequilibrium steady state where the system is subjected to an applied force as well as to a "Gaussian thermostat," which allows the system to reach a steady state by removing the heat produced by the applied force [2,3]. Here one can relate the transport coefficients to the change in the sum of all, or in some cases, a pair [2], of the Lyapunov exponents in the steady state.

The system considered here is a dilute, thermostated two-dimensional Lorentz gas, where a particle with charge $q$ and mass $m$ moves in an infinite random array of fixed hard disk scatterers of radius $a$ and density $n, n a^{2} \ll 1$, subject to a constant, uniform, external field $\vec{E}$ as well as to a frictional thermostat which maintains the speed of the particle at a constant value $v$. The system approaches a nonequilibrium steady state, characterized by an attractor in phase space. In this steady state, the following relation holds for the macroscopic diffusion coefficient $D$ for the particle [or, equivalently, the conductivity $\sigma$ related to $D$ by an Einstein formula $\left.D=m(v / q)^{2} \sigma\right]$ and the Lyapunov exponents $\lambda_{+}(\varepsilon)$ and $\lambda_{-}(\varepsilon)[2,4]: D=$ $-\lim _{\varepsilon \rightarrow 0}\left\{v^{2}\left[\lambda_{+}(\varepsilon)+\lambda_{-}(\varepsilon)\right]\right\} \varepsilon^{-2}$, where $\varepsilon=(q E / v m)$. Here we report the first theoretical calculation of both $\lambda_{+}(\varepsilon)$ and $\lambda_{-}(\varepsilon)$, for this system, using kinetic theory. We also present a comparison with extensive computer simula- tions, as well as with the general relation, given above. We apply a method based on the extended Lorentz-Boltzmann (LB) equation developed by van Beijeren and Dorfman for computing the Lyapunov exponents $[5,6]$.

The motion of the particle is described by a position vector $\vec{r}$, and velocity $\vec{v}$. Between collisions with the disks, the (non-Hamiltonian) equations of motion of the particle in the field and with an energy conserving Gaussian thermostat are

$$
\begin{gathered}
\dot{x}=v_{x}=v \cos \theta, \quad \dot{y}=v_{y}=v \sin \theta, \\
\dot{v}_{x}=v \varepsilon-\alpha v_{x}, \quad \dot{v}_{y}=-\alpha v_{y} .
\end{gathered}
$$

Here $\theta$ is the angle the particle's velocity makes with the applied field, in the $x$ direction, and $\alpha=v_{x} \varepsilon / v$ represents the strength of the frictional force provided by the Gaussian thermostat [2], determined by the condition that the kinetic energy remains constant.

The instantaneous change in velocity of the moving particle upon collisions is $\vec{v}^{\prime}-\vec{v}=-2 \hat{k}(\vec{v} \cdot \hat{k})$, where $\vec{v}^{\prime}$ is its velocity after collision, and $\hat{k}$ is the unit vector in the direction from the center of the scatterer to the point of impact.

To determine the positive Lyapunov exponent $\lambda_{+}$we first consider the separation of two diverging trajectories that start simultaneously at the same initial spatial point, but have slightly different initial velocity directions, specified by angles $\theta$ and $\theta^{\prime}$, with $\theta(t=0)=\theta_{0}$ and $\theta^{\prime}(t=$ $0)=\theta_{0}+\delta \theta_{0}$. We choose to measure the separation of trajectories along a line which is at all times $t>0$ perpendicular to the reference trajectory. Because the trajectories are curved by the thermostated field, the intersection of this line with the adjacent trajectory does not occur at the point that a particle following the adjacent trajectory will reach at time $t$, even to first order in the infinitesimal quantities, such as $\delta \theta_{0}$. In order to determine the Lyapunov exponents, one must take effects produced by the curving of the trajectories into account (see Fig. 1). The 


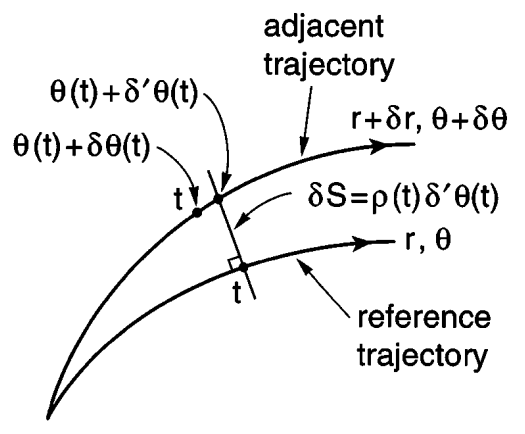

FIG. 1. Geometry for the curvature $\rho(t)$ in the applied field.

separation between the two trajectories at time $t, \delta S(t)$, can be written as the product of a radius of curvature $\rho(t)$ and $\delta^{\prime} \theta(t)$, the difference between the velocity angles of the two trajectories at the points of intersection with the perpendicular line, or $\rho(t)=S(t) / \delta^{\prime} \theta(t)$. The radius of curvature changes continuously between collisions, and instantaneously at the collisions of the particle with the scatterers. Simple geometric considerations yield an equation for the rate of change of $\rho(t)$ between collisions as

$$
\dot{\rho}=v+(\rho \varepsilon) \cos \theta+\frac{\rho^{2} \varepsilon^{2}}{v} \sin ^{2} \theta .
$$

The instantaneous change in the radius of curvature due to a collision is given by [7]

$$
\frac{1}{\rho_{+}}=\frac{1}{\rho_{-}}+\frac{2}{a \cos \phi},
$$

where $\vec{v} \cdot \hat{k}=-\cos \phi, \phi$ is the angle of incidence at collision, and $\rho_{-}$and $\rho_{+}$are the radii of curvature before and after collision, respectively.

We now compute the positive Lyapunov exponent $\lambda_{+}$ from the rate of separation of diverging trajectories. The result of Sinai [7] for the positive Lyapunov exponent in a field-free Lorentz gas can be straightforwardly generalized to include the case under discussion:

$$
\lambda_{+}=\lim _{T \rightarrow \infty} \frac{1}{T} \int_{t_{0}}^{t_{0}+T} \frac{v}{\rho(t)} d t=\left\langle\frac{v}{\rho}\right\rangle_{\text {s.s. }} .
$$

Here we assume ergodicity and calculate the ensemble average in the steady state, denoted by the angular brackets with subscripts s.s., using a nonequilibrium steady state distribution function for the moving particle in the constant thermostated applied field. The main idea is to consider an ensemble of similarly prepared systems, assume that the distribution function for the moving particle, in this ensemble, reaches a spatially homogeneous steady state (since there is no way to distinguish one spatial point from another in the ensemble average), and to derive and solve a LB equation for the distribution function for the moving particle $f(\vec{v}, \rho)$, where the variables include both the velocity and the radius of curvature describing the separation of trajectories of the moving particle and an adjacent trajectory, as described above.

Thus we write

$$
\lambda_{+}=\int d \vec{v} \int_{0}^{\infty} d \rho \frac{v}{\rho} f(\vec{v}, \rho)
$$

assuming that $f$ has been normalized to unity. The LB equation for $f$ is

$$
\nabla_{\vec{v}} \cdot(f \dot{\vec{v}})+\frac{\partial}{\partial \rho}(\dot{\rho} f)=\left(\frac{\partial f}{\partial t}\right)_{\mathrm{coll}} .
$$

Since the dynamics between collisions is not Hamiltonian, the usual form of the streaming terms on the left hand side of the LB equation must be replaced by the form that reflects the total conservation of particles under the actual, non-Hamiltonian dynamics. The left hand side of the LB equation can be obtained from the equations of motion, Eq. (1), and Eq. (2) for $\dot{\rho}$

$$
-\varepsilon \frac{\partial}{\partial \theta}(f \sin \theta)+\frac{\partial}{\partial \rho}\left[\left(v+\rho \varepsilon \cos \theta+\frac{\rho^{2} \varepsilon^{2}}{v} \sin ^{2} \theta\right) f\right]=\left(\frac{\partial f}{\partial t}\right)_{\text {coll }},
$$

where we used the constant speed of the particle to denote $f(\vec{v}, \rho)$ by $f(\theta, \rho)$. The right hand side (rhs) of the LB equation is the change in $f$ due to collisions given previously [5] as

$$
\left(\frac{\partial f}{\partial t}\right)_{\text {coll }}=n a v \int_{-\pi / 2}^{\pi / 2} d \phi \cos \phi \int_{0}^{\infty} d \rho^{\prime} \delta\left(\rho-\frac{(a \cos \phi) / 2}{1+(a \cos \phi) / 2 \rho^{\prime}}\right) f\left(\vec{v}^{\prime}, \rho^{\prime}\right)-2 \operatorname{nav} f(\vec{v}, \rho) .
$$

To leading order in the density, the collision terms can be simplified further by approximating the factor $1+$ $(a \cos \phi) / 2 \rho^{\prime}$ inside the $\delta$ function by unity since $\rho^{\prime}$ is typically on the order of the mean free path, so that $\rho^{\prime} \gg a$. Then Eq. (7) can be solved by expanding $f$ as a power series in $\varepsilon$ and inserting the solution into Eq. (5). We obtain

$$
\lambda_{+}(\varepsilon)=\lambda_{0}-\frac{11}{48} t_{0} \varepsilon^{2}+O\left(\varepsilon^{4}\right)
$$

where $t_{0}=\ell / v$, with $\ell=(2 n a)^{-1}$ the mean free path of the moving particle, and $\lambda_{0}$ is given in Ref. [5].
The calculation of $\lambda_{-}(\varepsilon)$ requires the study of trajectories that asymptotically converge to the reference trajectory of the moving particle rather than diverge from it. That is, the negative Lyapunov exponent can only be determined if one can find trajectories that lie on the stable manifold of the reference trajectory, which is typically difficult since almost all of the adjacent trajectories will eventually diverge from it. To overcome this difficulty we consider the time-reversed motion of the moving particle [8]. This allows us to consider diverging trajectories again. We thus consider the steady state distribution func- 
tion $f_{-}(\vec{v}, \rho)$ for trajectories of the particle with velocity $\vec{v}$ and radius of curvature $\rho$ on the time reversal of the stable manifold. The equation for $f$ - takes the form of an "anti-Lorentz-Boltzmann" (ALB) equation. This unusual form is dictated by the observation that if the moving particle and the scatterer with which it collides are uncorrelated before collision in the forward motion, then in the time-reversed motion the moving particle and scatterer will be uncorrelated after the collision. That is, to obtain the ALB, one must use the Stosszahlansatz for the exiting collision cylinders, rather than for those before the collision [6,9]. The ALB then reads

$$
-\varepsilon \frac{\partial}{\partial \theta}\left(f_{-} \sin \theta\right)+\frac{\partial}{\partial \rho}\left[\left(v+\rho \varepsilon \cos \theta+\frac{\rho^{2} \varepsilon^{2}}{v} \sin ^{2} \theta\right) f_{-}\right]=\left(\frac{\partial f_{-}}{\partial t}\right)_{\text {coll }},
$$

where

$$
\begin{aligned}
\left(\frac{\partial f_{-}}{\partial t}\right)_{\text {coll }}= & n a v \int_{-\pi / 2}^{\pi / 2} d \phi \cos \phi \int_{0}^{\infty} d \rho^{\prime} \delta(\rho-(a \cos \phi) / 2) f_{-}\left(\vec{v}, \rho^{\prime}\right)-n a v \\
& \times \int_{-\pi / 2}^{\pi / 2} d \phi \cos \phi \frac{\int_{0}^{\infty} d \rho^{\prime} f_{-}\left(\vec{v}^{\prime}, \rho^{\prime}\right)}{\int_{0}^{\infty} d \rho^{\prime} f_{-}\left(\vec{v}, \rho^{\prime}\right)} f_{-}(\vec{v}, \rho) .
\end{aligned}
$$

The first term on the rhs of Eq. (11) is the gain term. It is constructed by (a) using the Stosszahlansatz for the collision cylinders centered on particles which are produced in collisions with velocity $\vec{v}$, (b) requiring that the radius of curvature after collision be $\rho$, and (c) noting that before collision the radius of curvature will typically be on the order of a mean free path, so that after collision $\rho$ will be very close to $(a / 2) \cos \theta$. The loss term in Eq. (11) can be obtained by noting that (a) the rate at which particles with velocity $\vec{v}$ disappear due to collisions with angle of incidence $\phi$ is $n a v \cos \phi \int_{0}^{\infty} d \rho^{\prime} f_{-}\left(\vec{v}^{\prime}, \rho^{\prime}\right)$, and (b) the fraction of those which disappear having radius of curvature $\rho$ is $f_{-}(\vec{v}, \rho)\left[\int_{0}^{\infty} d \rho^{\prime} f_{-}\left(\vec{v}, \rho^{\prime}\right)\right]^{-1}$. These considerations lead directly to the rhs of Eq. (11), and a more detailed explanation will be given elsewhere [6].

Equation (10) can be solved by imposing the requirement that

$$
\int d \rho f_{-}(\vec{v}, \rho)=f_{-}(\vec{v})
$$

with $f_{-}(\vec{v})$ the time-reversed steady state solution of the spatially homogeneous Lorentz-Boltzmann equation. To understand this condition, we note that when Eq. (10) is integrated over all values of $\rho$ to obtain an equation for the velocity distribution function $f_{-}(\vec{v})$ one obtains an ALB equation with a collision operator that has the opposite sign from the usual Lorentz-Boltzmann equation, due to the fact that we are considering the time-reversed motion, and using the Stosszahlansatz after collisions [9]. For consistency, we then require that the steady state solutions of the $\rho$ integrated LB and ALB equations be related by a simple time-reversal operation, as indicated by Eq. (12).

The negative Lyapunov exponent is obtained from $f_{-}$ by

$$
\lambda_{-}=-\int d \vec{v} \int_{0}^{\infty} d \rho \frac{v}{\rho} f_{-}(\vec{v}, \rho)
$$

with $f$ - normalized to unity. The negative sign on the rhs of Eq. (13) is the result of the time-reversal procedure. Again, the equation for $f_{-}$can be solved by expanding $f_{-}$in powers of $\varepsilon$. We finally obtain

$$
\lambda_{-}=-\lambda_{0}-\frac{7}{48} t_{0} \varepsilon^{2}+O\left(\varepsilon^{4}\right) .
$$

Using the relation between the diffusion coefficient $D$ and the sum of the Lyapunov exponents discussed earlier, we recover the correct low density value $D=(3 / 8) t_{0} v^{2}$.

It is important to compare these results with those obtained from computer simulations of the same system. For this purpose we distributed $10^{5}$ nonoverlapping scatterers randomly in a square simulation cell with periodic boundaries. Between collisions the trajectory of the moving particle is computed from an analytical solution [10] of the thermostated equations of motion. The collision points with the scatterers are determined numerically with an accuracy of $10^{-12}$. The Lyapunov exponents were computed in tangent space by a simultaneous integration of the linearized equations of motion for the intercollisional streaming and an exact linearization of the map which relates the separation of trajectories after collision to that before collision [11]. In Fig. 2 we show the deviation of the nonvanishing Lyapunov exponents from their equilibrium values as a function of the squared applied field. All numbers are made dimensionless by using $a, v, m$, and $q$ as the respective units for length, velocity, mass, and charge. Two reduced scatterer densities $n^{*}=n a^{2}$ were considered, 0.001 (circles) and 0.002 (squares). Each point is obtained by averaging over ten simulation runs with different scatterer configurations and a total of $4 \times 10^{6}$ collisions for each run. The standard deviation for the exponents is $\sim 0.1 \%$. The lines refer to the theoretical expressions, Eqs. (9) and (14). Both $\lambda_{+}$and $\lambda_{-}$exhibit the predicted quadratic weak field behavior. These results are clearly consistent with the theoretical predictions for the field-dependent Lyapunov exponents, though at densities somewhat higher 


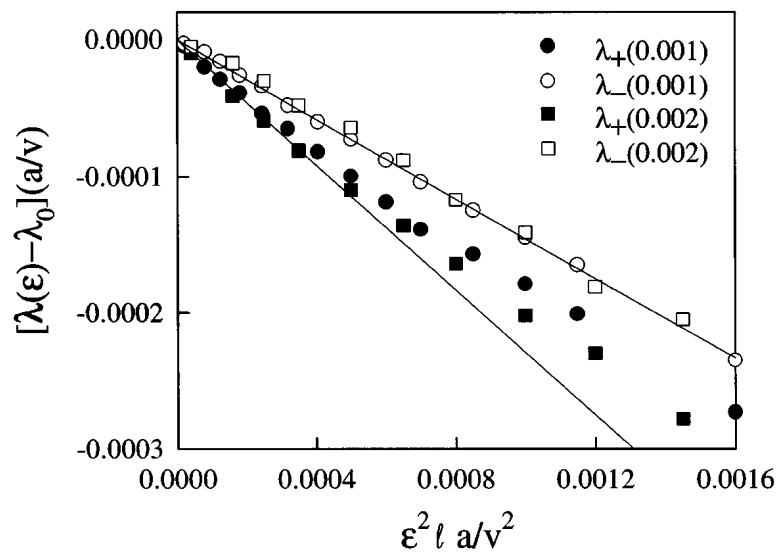

FIG. 2. Field dependence of the deviation of the Lyapunov exponents from their equilibrium value for the reduced densities $n^{*}=0.001$ and $n^{*}=0.002$. $\varepsilon=q E / v m$, where $E$ is the applied field. $\ell$ is the mean free path, and $q, m$, and $v$ are the charge, mass, and velocity of the particle, respectively. $a$ is the diameter of the scatterers.

than those for which the LB results hold without density corrections. Figure 3 shows $D /(\ell v)$ as a function of $\varepsilon^{2} \ell a / v^{2}$ for $n^{*}=0.001$ (full circles) and 0.002 (open circles). The diffusion coefficient $D$ is obtained from the conductivity $\sigma$ through the Einstein formula, and $\sigma$ is numerically computed from $\sigma=\left\langle q v_{x}\right\rangle_{\mathrm{s} \text { s. }} / E$, the ratio of the time-averaged dissipative current to the applied field. Alternatively, $D$ can be obtained also from the Lyapunov exponents as indicated above. On the scale of Fig. 3 both methods yield indistinguishable results.

We mention here that to obtain agreement between the simulation data for $D$ and theory it was necessary to add to the low-density Lorentz-Boltzmann result $(3 / 8) \ell v$, the dotted top horizontal line in Fig. 3, known [12] moderate density corrections, which include essential contributions from logarithmic terms of the form $n^{*} \ln n^{*}+O\left(n^{*}\right)$. The numerical nonequilibrium results converge well to

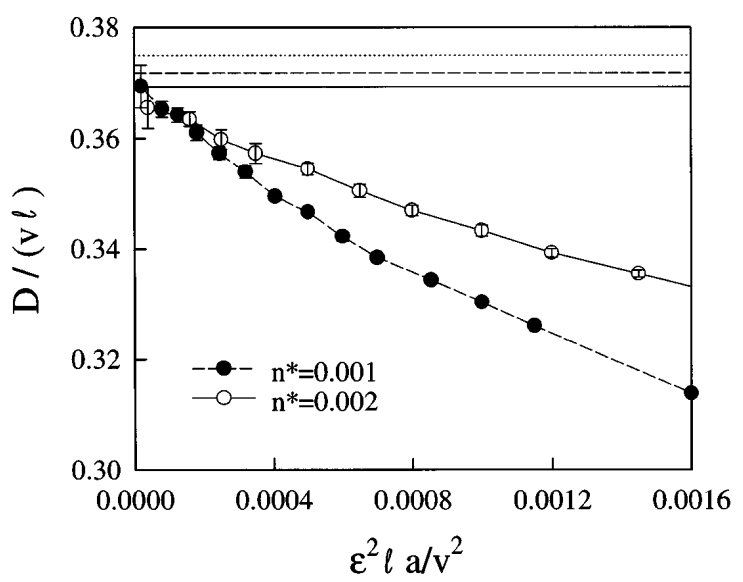

FIG. 3. Dependence of the diffusion coefficient $D$ on the field for the two densities $n^{*}=0.001$ and 0.002. All other quantities are as in Fig. 2. The horizontal lines are explained in the text. these corrected values for vanishing field indicated by the dashed $\left(n^{*}=0.001\right)$ and solid $\left(n^{*}=0.002\right)$ horizontal lines. Thus the computer results confirm the presence of these nonanalytic terms in the density expansion.

As a check we computed the Lyapunov exponents for still smaller densities, $n^{*}=0.0001$, and fields $<10^{-5}$ with a very efficient direct-simulation Monte Carlo method [13] and found perfect agreement with our other simulation results quoted above.

We conclude by remarking that this work illustrates the power of kinetic theory methods for computing quantities of interest to both statistical mechanics and dynamical systems theory $[5,6,14]$.

J.R.D. thanks A. Latz for helpful discussions, and the National Science Foundation for support under Grant No. PHY-93-21312. H. A.P. and Ch. D. gratefully acknowledge support from the Fonds zur Förderung der wissenschaftlichen Forschung, Grant No. P09677, and the generous allocation of computer resources by the Computer Center of the University of Vienna. E. G.D.C. is indebted to the U.S. Department of Energy for support under Grant No. DE-FG 02-88-ER 13847.

[1] P. Gaspard and G. Nicolis, Phys. Rev. Lett. 65, 1693 (1990); P. Gaspard and J. R. Dorfman, Phys. Rev. E 52, 3525 (1995).

[2] D. J. Evans and G.P. Morriss, Statistical Mechanics of Nonequilibrium Liquids (Academic Press, London, 1990); D. J. Evans, E. G. D. Cohen, and G. P. Morriss, Phys. Rev. A 42, 5990 (1990).

[3] W. G. Hoover, Computational Statistical Mechanics (Elsevier Publ. Co., Amsterdam, 1991); B. L. Holian, W. G. Hoover, and H. A. Posch, Phys. Rev. Lett. 59, 10 (1987); H. A. Posch and W. G. Hoover, Phys. Rev. A 38, 473 (1988); 39, 2175 (1989); W. N. Vance, Phys. Rev. Lett. 69, 1356 (1992).

[4] N. I. Chernov, G. L. Eyink, J. L. Lebowitz, and Ya.G. Sinai, Commun. Math. Phys. 154, 569 (1993).

[5] H. van Beijeren and J. R. Dorfman, Phys. Rev. Lett. 74, 4412 (1995).

[6] A. Latz, H. van Beijeren, and J.R. Dorfman (unpublished).

[7] Ya. G. Sinai, Russ. Math. Surv. 25, 137 (1970).

[8] Note the same is done in computer simulations designed for measuring $\lambda_{-}$; cf. Refs. [2,3] above.

[9] E. G. D. Cohen and T. H. Berlin, Physica (Utrecht) 26, 717 (1960).

[10] B. Moran and W. G. Hoover, J. Stat. Phys. 48, 709 (1987).

[11] Ch. Dellago, L. Glatz, and H. A. Posch, Phys. Rev. E 52, 4817 (1995); Ch. Dellago, H. A. Posch, and W. G. Hoover, Phys. Rev. E 53, 1485 (1996).

[12] C. Bruin, Physica (Utrecht) 72, 261 (1974).

[13] Ch. Dellago and H. A. Posch (unpublished).

[14] E. G. D. Cohen, in "Boltzmann and Statistical Mechanics" (Cambridge U. Press, Cambridge, England, to be published). 